\title{
Knockdown of lincRNA PADNA promotes bupivacaine-induced neurotoxicity by miR- 194/FBXW7 axis
}

\author{
Fan Yuning ${ }^{1}$, Chen Liang ${ }^{2}$, Wang Tenghuan ${ }^{1}$, Nan Zhenhua ${ }^{1}$ and Gong Shengkai ${ }^{1 *}$ (D)
}

\begin{abstract}
Background: The aim of the study was to explore the function and mechanism of lincRNA PADNA in bupivacaineinduced neurotoxicity.

Methods: Mouse DRG neurons were cultured in vitro and treated with bupivacaine to establish a neurotoxicity model. Caspase3 activity, cell viability, and TUNEL assays were analyzed to assess the role of lincRNA PADNA. A dual-luciferase reporter assay was used to determine the binding target of lincRNA PANDA.

Results: The expression of lincRNA PADNA was significantly increased with increasing concentrations of bupivacaine. Functional analysis revealed that knockdown of lincRNA PADNA increased caspase3 activity and inhibited cell viability. Western blot analysis showed that knockdown of lincRNA PADNA promoted cleaved caspase3 levels. We also revealed that lincRNA PADNA may bind with miR-194. Knockdown of miR-194 rescued the function of lincRNA PADNA, suggesting that lincRNA PADNA may sponge miR-194. In addition, we provided new evidence that the lincRNA PADNA/miR-194/FBXW7 axis plays an important role in the neurotoxicity process.

Conclusion: We performed comprehensive experiments to verify the function and mechanism of lincRNA PADNA in bupivacaine-induced neurotoxicity. Our study provides new evidence and clues for the prevention of neurotoxicity.
\end{abstract}

Keywords: lincRNA, PADNA, miR-194, FBXW7

\section{Introduction}

Bupivacaine is one of the most commonly used anesthetics for local infiltration anesthesia (Radwan et al. 2002; Guo et al. 2017; Chalkiadis et al. 2016). Studies have demonstrated that the adverse drug reactions (ADRs) to bupivacaine are mainly limited to the central nervous system (CNS) and cardiovascular system because of the systemic absorption of bupivacaine (Ammar and Mahmoud 2012; Kurihara et al. 2008). During the past few decades, bupivacaine has been found to be neurotoxic in the setting of local injection, causing symptoms such as paralysis, paresthesia, hypoventilation,

\footnotetext{
* Correspondence: gsk1972@sohu.com

'Department of Anesthesiology, Pain and Perioperative Medicine, The First Affiliated Hospital of Zhengzhou University, Henan, China

Full list of author information is available at the end of the article
}

and fecal and urinary incontinence (Xianjie et al. 2013; Traore et al. 2006; Helal et al. 2016; Ferrillo 2016). The side effects of bupivacaine have aroused enormous interest and attention, and a great number of studies have been conducted to elucidate the mechanism of bupivacaine-induced neurotoxicity and to find ways to prevent or target these side effects (Xianjie et al. 2013). However, the results have been difficult to interpret, and the mechanism of bupivacaine-induced neurotoxicity remains unclear.

Efforts have been made to investigate the association between bupivacaine-induced neurotoxicity and noncoding RNAs (ncRNAs) (Wang et al. 2015). NcRNAs are RNA molecules that cannot be translated into protein (Liu et al. 2016). There are different types of ncRNAs, including transfer RNAs, ribosomal RNAs, microRNAs,

(c) The Author(s). 2020 Open Access This article is licensed under a Creative Commons Attribution 4.0 International License, which permits use, sharing, adaptation, distribution and reproduction in any medium or format, as long as you give appropriate credit to the original author(s) and the source, provide a link to the Creative Commons licence, and indicate if changes were made. The images or other third party material in this article are included in the article's Creative Commons licence, unless indicated otherwise in a credit line to the material. If material is not included in the article's Creative Commons licence and your intended use is not permitted by statutory regulation or exceeds the permitted use, you will need to obtain permission directly from the copyright holder. To view a copy of this licence, visit http://creativecommons.org/licenses/by/4.0/. 
and lncRNAs (Yamada et al. 2018; Li et al. 2018). Jiang $\mathrm{R}$ found that miR-489-3p could promote bupivacaineinduced apoptosis by regulating the PI3K/AKT pathway (Jiang et al. 2017). LncRNAs are defined as transcripts with lengths exceeding 200 nucleotides (Song et al. 2017), which have been widely studied and found to be abundantly and functionally important in regulating the cell cycle (Liu et al. 2015), cell metabolism (Zhang et al. 2016a) and related diseases such as malignant tumors (Gu et al. 2018). However, the role of lncRNAs in bupivacaine-induced neurotoxicity has rarely been researched.

In the current research, we investigated the long noncoding RNA Gm14012 (named lincRNA PADNA, protect cell death RNA, for its role in protect against cell death). To investigate the underlying mechanism of how lincRNA PADNA participates in bupivacaine-induced neurotoxicity, we conducted bioinformatics analysis, and the results revealed that lincRNA PADNA played a protective role through inhibition of the progression of bupivacaine-induced neurotoxicity by sponging miR194, which has been reported to inhibit tumor progression (Wu et al. 2014). miR-194 was predicted to target the 3'UTR of the cancer-related protein F-box and WD repeat domain containing 7 (FBXW7) by analysis in StarBase2.0. The current research may provide new targets for inhibiting or reversing bupivacaine-induced neurotoxicity.

\section{Materials and methods}

\section{Cell culture and treatment}

HEK293 cells were stored in our laboratory, and primary dorsal root ganglion (DRG) neurons were isolated from 5-week-old C57BL/6 mice as previously described (Zhang et al. 2016b). Briefly, 5-week-old C57BL/6 mice were anesthetized and sacrificed by cervical dislocation. The L4-L5 portion of the spinal cord was extracted. The dorsal root ganglia were collected and dissociated with $0.25 \%$ trypsin (Invitrogen, USA). The cells were washed with $2.5 \%$ bovine serum albumin (BSA, Invitrogen, USA) and resuspended in serum-free neurobasal medium (Invitrogen, USA) supplemented with penicillin/ streptomycin (40,000 unit/L, Invitrogen, USA) and B27 serum-free supplement (Invitrogen, USA). To induce neurotoxicity, DRG neurons were treated with various concentrations of bupivacaine $(0.5,1.0,1.5$ or $2.0 \mathrm{mM}$ ) for $6 \mathrm{~h}, 12 \mathrm{~h}, 24 \mathrm{~h}$, and $48 \mathrm{~h}$.

\section{Transfection}

The knockdown vectors of lincRNA PADNA were constructed by Gene Pharma (Shanghai, China). Empty vectors and vectors with wild-type (WT) or mutant (mut) binding sites for miR-194 were constructed by Gene Pharma (Shanghai, China). The 3'-untranslated region
(UTR) of FBXW7, containing wild-type (WT) or mutant (mut) binding sites for miR-194, was amplified and cloned into the pGL3 vector (Promega, Madison, WI) to generate the vector pGL3-WT-FBXW7-3'-UTR or pGL3-mut-FBXW7-3'-UTR. The miR-194 mimic, miR194 inhibitor, mimic NC, and inhibitor NC were purchased from Shanghai Gene Pharma (Shanghai, China). Briefly, DRG neurons were cotransfected with vectors, miR-194 mimic, miR-194 inhibitor, mimic NC and inhibitor NC by the Lipo3000 reagent (Invitrogen) according to the manufacturer's protocol. Cells were incubated for $48 \mathrm{~h}$ before further research.

\section{Cell viability assay}

DRG neurons were seeded into 96-well plates and treated with bupivacaine $(0.5,1.0,1.5$ or $2.0 \mathrm{mM})$ for 24 $\mathrm{h}$ to establish a bupivacaine-induced neurotoxicity model. Ten microliters of 3-(4,5-dimethylthiazol-2-yl)-2, 5-diphenyltetrazolium bromide (MTT) solution $(5 \mathrm{mg} /$ $\mathrm{mL}$, Beyotime, Shanghai, China) was added to each well and incubated for $4 \mathrm{~h}$ at $37^{\circ} \mathrm{C}$. Cell viability was measured by a microplate reader at $570 \mathrm{~nm}$ (Bio-Tek, Winooski, VT).

\section{Caspase-3 activity}

A caspase-3 activity assay kit (Beyotime, Shanghai, China) was used to assess caspase- 3 activity according to the manufacturer's protocol. DRG neurons were lysed and centrifuged. A final concentration of $0.2 \mathrm{mM}$ ADEV D-pNA (caspase-3 substrate) was added to the cell supernatant and incubated for $1 \mathrm{~h}$. Caspase- 3 activity was measured by a microplate reader at $405 \mathrm{~nm}$ (BioTek, Winooski, VT).

\section{TUNEL assay}

A terminal deoxynucleotidyl transferase-mediated dUTP nick end-labeling (TUNEL) assay was conducted to evaluate the apoptosis of DRG neurons. DRG neurons were incubated with $\mathrm{TdT}$ and fluorescein-labeled dUTP for $45 \mathrm{~min}$ at $37^{\circ} \mathrm{C}$. Then, a FACSCalibur flow cytometer was used to measure the percentage of apoptotic cells.

\section{Dual-luciferase reporter assay}

Empty vector or vector with the wild-type (WT) or mutant (mut) binding sites for miR-194 was cotransfected with the miR-194 mimic or mimic NC. Luciferase activity was analyzed using a dual-luciferase reporter system following the manufacturer's protocol. Firefly luciferase activity and Renilla luciferase activity were measured with Multiskan Spectrum (Thermo Fisher, USA). Similarly, empty vector or vector containing the 3 '-UTR of FBXW7 with the wild-type or mutant binding sites for miR-194 was cotransfected with the miR-194 mimic or mimic NC. Luciferase activity was analyzed using a dual- 
luciferase reporter system. Firefly luciferase activity and Renilla luciferase activity were measured with Multiskan Spectrum (Thermo Fisher, USA).

\section{Real-time PCR}

Total RNA was extracted by TRIzol reagent (Thermo Fisher Scientific). RNA reverse transcription was performed using a PrimeScript ${ }^{\mathrm{tm}}$ RT reagent Kit with gDNA eraser (RR047A; Takara, Tokyo, Japan), and cDNA was assayed using SYBR ${ }^{\circ}$ Premix Ex Taq ${ }^{\text {Tn }}$ (RR420A; Takara, Tokyo, Japan). The data were normalized to $\beta$-actin levels and further analyzed by the $2^{-\Delta \Delta C T}$ method.

\section{Western blotting}

DRG neurons were harvested and lysed by RIPA lysis buffer containing proteinase inhibitor (Roche, USA). Total protein was quantified using a BCA protein assay kit (Pierce, Rockford, IL, USA). Protein samples were resolved by $10 \%$ SDS-PAGE gels and transferred to polyvinylidene difluoride membranes. After blocking, the membranes were incubated with primary antibodies against caspase3 (1:1000, Abcam, MA, USA), FBXW7 (1:1000, Abcam, MA, USA) and actin (1:1000, Abcam, MA, USA) at $4{ }^{\circ} \mathrm{C}$ overnight, followed by incubation with a peroxidase-conjugated goat anti-rabbit (or mouse) IgG antibody. Immunopositive bands were analyzed using a FluorChem M system (ProteinSimple, San Jose, CA, USA).

\section{Data analysis}

We used SPSS 23.0 to calculate the data values (means \pm standard error of the mean). Statistical differences were analyzed by unpaired 2-sided Student's $t$ test or 1-way ANOVA with Bonferroni correction for multiple comparisons. The statistical significance was set at $P<0.05$.

\section{Results}

The establishment of a bupivacaine-induced neurotoxicity model

To analyze the bupivacaine-induced neurotoxicity process, we first verified the model. Apoptosis and cell viability were analyzed via caspase 3 activity and MTT assays. Caspase 3 activity increased with increasing concentrations of bupivacaine and reached its highest level at $2.0 \mathrm{mM}$, the relative increase was about $1.01 \pm 0.1$, $1.79 \pm 0.08,1.89 \pm 0.05,2.72 \pm 0.08,3.11 \pm 0.07$ (Fig. 1a). Cell viability was significantly reduced compared with that in the control group. In addition, $2.0 \mathrm{mM}$ bupivacaine reduced cell viability by almost $50 \%$, the relative decrease was about $1.00 \pm 0.1,0.82 \pm 0.08,0.75 \pm 0.09$, $0.63 \pm 0.07,0.53 \pm 0.08$ (Fig. 1b). Thus, our results confirmed that bupivacaine induced cell apoptosis.

\section{Expression of lincRNA Gm14012 in bupivacaine-induced neurotoxicity}

We first detected the expression of lincRNA Gm14012 to investigate the possible change. As shown in Fig. 1c, the expression of lincRNA Gm14012 increased with increasing bupivacaine concentration and reached a maximum of an almost 16 -fold increase at $2.0 \mathrm{mM}$, the relative increase was about $1.03 \pm 0.1,1.92 \pm 0.18,4.13 \pm$ $0.24,8.92 \pm 0.28,17.05 \pm 0.27$. Similarly, we also detected the expression of lincRNA Gm14012 after exposure to $1.0 \mathrm{mM}$ for different durations. Our results showed that the expression of lincRNA Gm14012 increased with time, the relative increase was $1.01 \pm 0.12,1.33 \pm 0.19$, $1.87 \pm 0.2,3.77 \pm 0.26,7.09 \pm 0.3$ (Fig. $1 \mathrm{~d}$ ).

\section{Function of lincRNA PADNA in bupivacaine-induced neurotoxicity}

We constructed a knockdown vector to assess the function of lincRNA Gm14012. As shown in Fig. 2a, the expression of lincRNA Gm14012 was significantly downregulated, the relative decrease was about 0.66 , 0.48. Next, we used the MTT assay to assess the

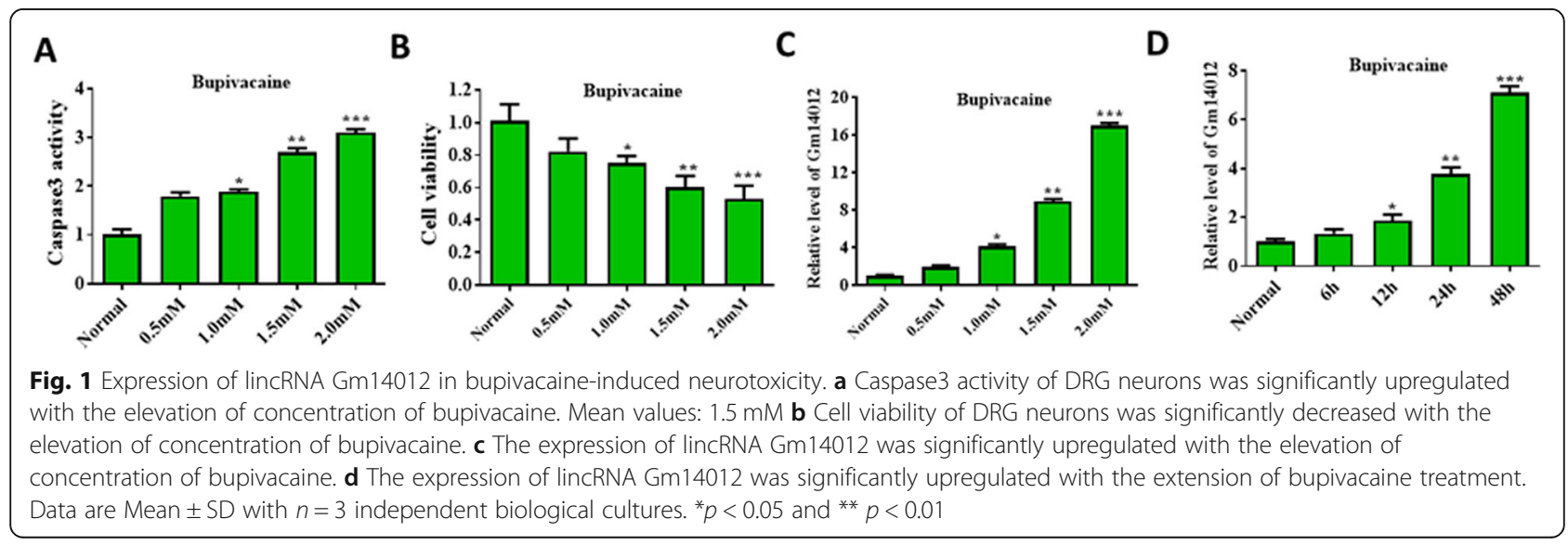




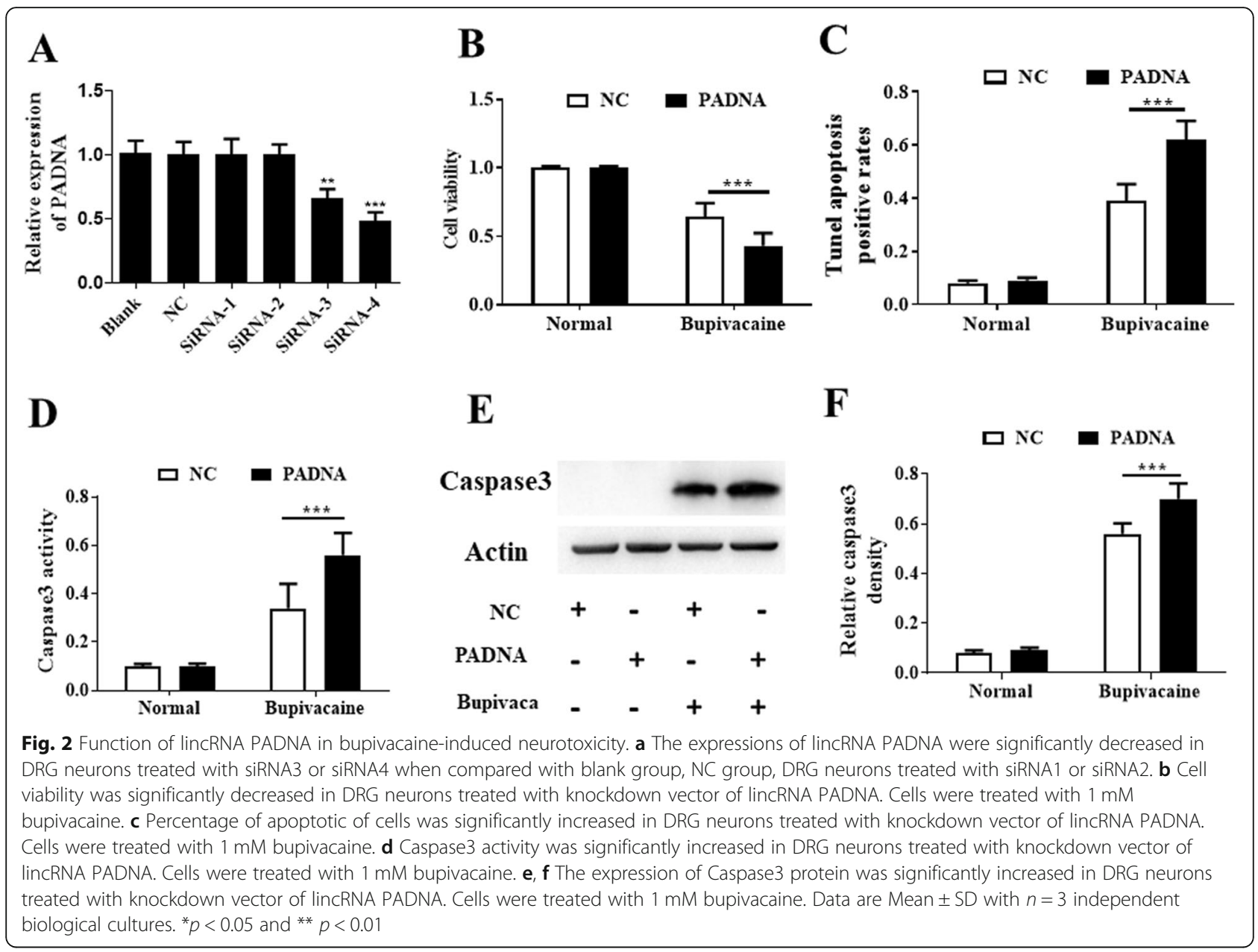

function of lincRNA Gm14012. Interestingly, knockdown of lincRNA Gm14012 significantly promoted cell death and reduced cell viability after exposure to bupivacaine, the relative decrease was about $0.64 \pm 0.1,0.43 \pm$ 0.08 (Fig. 2b). To date, there has been no report on lincRNA Gm14012 in bupivacaine-induced neurotoxicity. Thus, we named this lincRNA PADNA (protect cell death RNA). To further explore the function of lincRNA PADNA in bupivacaine-induced neurotoxicity, we performed comprehensive analyses, such as TUNEL assay, caspase 3 activity assay, and western blotting. TUNEL assays were used to assess cell apoptosis. There was no significant difference between the two groups under normal conditions; however, when cells were exposed to $1.0 \mathrm{mM}$ bupivacaine, knockdown of lincRNA PADNA significantly increased the cell apoptosis rates, suggesting that knockdown of lincRNA PADNA promoted cell death, the relative increase was about $0.41 \pm$ $0.07,0.63 \pm 0.06$ (Fig. 2c). In addition, we measured caspase3 activity using a kit. Our results suggested that downregulation of lincRNA PADNA significantly increased caspase 3 activity, the relative increase was about
$0.34 \pm 0.09,0.56 \pm 0.08$ (Fig. $2 d$ ). Similar results were obtained from western blotting (Fig. 2e). Overall, our experiments demonstrated that lincRNA PADNA played a protective role in bupivacaine-induced neurotoxicity.

Preliminary analysis of the mechanism of lincRNA PADNA Long noncoding RNAs often act as sponge RNAs to bind with miRNAs to mediate different functions in numerous processes. To investigate the possible mechanism of lincRNA PADNA, we first used miRDNA to predict the potential binding targets of lincRNA PADN A. As shown in Fig. 3a, miR-194 was predicted to bind with lincRNA PADNA. Next, we used dual-luciferase reporter assays to verify whether lincRNA PADNA can bind with miR-194. Our results suggested that the relative luciferase activity of miR-194 was significantly reduced in the wt-lincRNA PADNA group, while no significant difference was detected in the mut-lincRNA PADNA group, the relative decrease was about $1.0 \pm 0.1$, $0.34 \pm 0.05$ (Fig. 3b). Thus, the above results preliminarily identified miR-194 as a target of lincRNA PADNA. Next, we detected the expression of lincRNA PADNA 


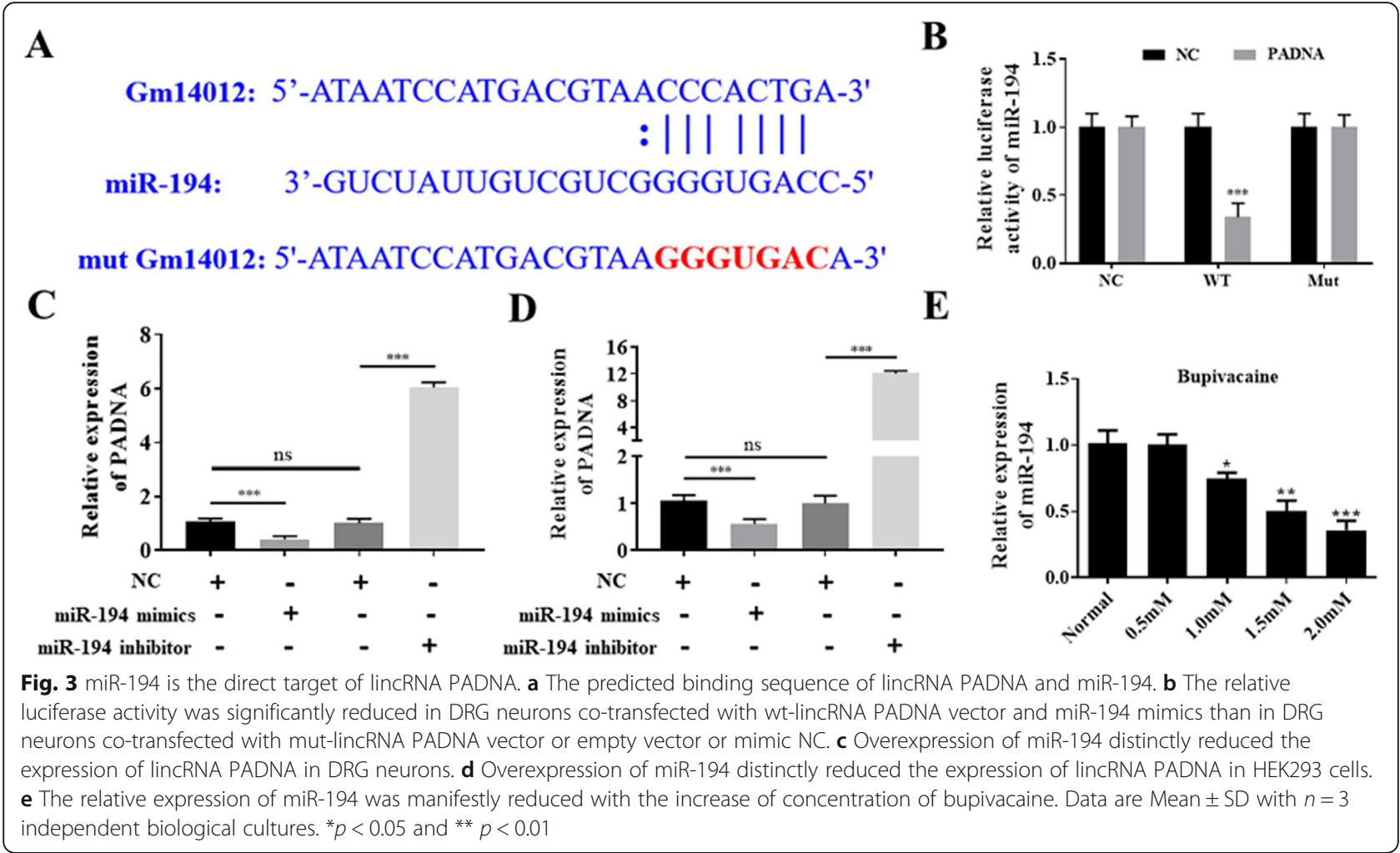

under different conditions. Overexpression of miR-194 markedly reduced the expression of lincRNA PADNA and knockdown of miR-194 increased the expression of PADNA, the relative values are $1.05 \pm 0.08,0.41 \pm 0.04$, $1.00 \pm 0.09,6.04 \pm 0.13$ (Fig. 3c). Similar results were also obtained in HEK293 cells, the relative values are $1.02 \pm$ $0.1,0.56 \pm 0.04,1.03 \pm 0.08,12.04 \pm 0.21$ (Fig. 3d). Moreover, we also analyzed the expression of miR-194 in bupivacaine-treated cells. Our results revealed that the expression of miR-194 was clearly reduced with increasing concentrations of bupivacaine and reached its lowest level at $2.0 \mathrm{mM}$ bupivacaine, the relative decrease was about $1.01 \pm 0.07, \quad 1.00 \pm 0.07,0.75 \pm 0.06, \quad 0.51 \pm 0.05$, $0.36 \pm 0.06$ (Fig. 3e). The above results revealed that lincRNA PADNA could negatively regulate the expression of miR-194.

\section{FBXW7 is the direct target of miR-194}

To further study the mechanism of miR-194 involved in bupivacaine-induced neurotoxicity, we used StarBase2.0 to predict the target of miR-194. We found that FBXW7 was predicted as the direct target of miR-194. Previous studies have also shown that FBXW7 is regulated by miR-194. However, the role of miR-194 and FBXW7 in bupivacaine-induced neurotoxicity remains unknown. Thus, we performed a comprehensive analysis to analyze their relationship. The binding sequence is shown in Fig. 4a. A dual-luciferase reporter assay revealed that the relative luciferase activity of wt-FBXW7 was significantly reduced in the miR-194 group, the relative values are $1.0 \pm 0.09,0.2 \pm 0.04$ (Fig. 4b). Overexpression of miR-194 markedly reduced the expression of FBXW7 and while silencing miR-194 reduced its expression, the relative values are $1.00 \pm 0.05,0.32 \pm$ $0.03,1.00 \pm 0.1,3.41 \pm 0.35$ (Fig. 4c). We also performed a western blot assay to analyze the protein level of FBXW7. Our results suggested that the protein level of FBXW7 was decreased in the miR-194 overexpression group (Fig. 4d). Figure 4e shows the negative correlation between miR-194 and FBXW7. In addition, we also analyzed the expression of FBXW7 in bupivacaine-treated cells. The expression of FBXW7 was increased with the increase in concentration and time course of bupivacaine and reached the highest level at $2.0 \mathrm{mM}$ bupivacaine, the relative values are $1.01 \pm 0.12, \quad 1.06 \pm 0.11, \quad 1.00 \pm 0.1, \quad 1.82 \pm$ $0.13,2.83 \pm 0.17$ (Fig. 4f, g). Thus, our study provides evidence that miR-194 negatively regulates the expression of FBXW7.

The lincRNA PADNA/miR-194/FBXW7 axis in bupivacaineinduced neurotoxicity

We performed comprehensive analysis to further verify the lincRNA PADNA/miR-194/FBXW7 pathway. The expression of FBXW7 was downregulated in the lincRNA PADNA knockdown group, as demonstrated 


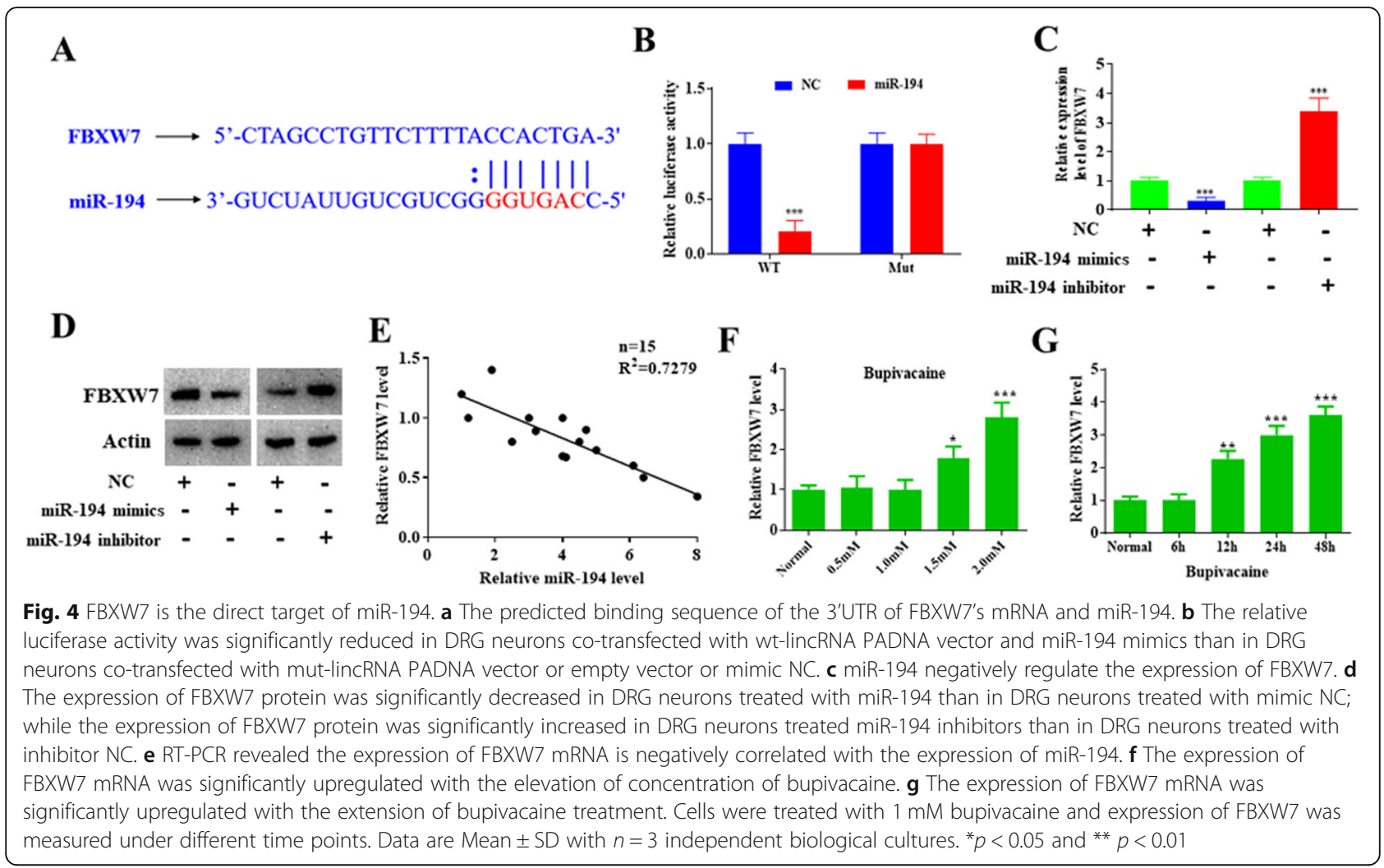

by $\mathrm{qPCR}$, the relative values are $1.01 \pm 0.1,0.31 \pm 0.07$ (Fig. 5a). Similar results were obtained by western blotting suggesting that lincRNA PADNA positively regulates FBXW7 (Fig. 5b). Considering the above results, we knocked down miR-194 and PADNA and then detected the expression of FBXW7. Interestingly, the expression of FBXW7 was increased in the cotransfection group as demonstrated qPCR and western blot assays, the relative values are $1.00 \pm 0.1$, $0.35 \pm 0.06,0.95 \pm 0.07$ (Fig. $5 \mathrm{c}, \mathrm{d}$ ). Thus, knockdown of miR-194 could block the effect of lincRNA PADN A. Therefore, a rescue experiment was performed to verify the relationship between miR-194 and FBXW7. TUNEL assays revealed that cotransfection of miR194 and PADNA could reduce the apoptotic effect compared with knockdown of lincRNA PADNA, the relative values are $0.11 \pm 0.01,0.35 \pm 0.04,0.6 \pm 0.06$, $0.41 \pm 0.04$ (Fig. 5e). In addition, caspase 3 activity and cleaved caspase3 levels were decreased, the relative values are $0.11 \pm 0.02,0.46 \pm 0.04,0.61 \pm 0.06,0.45 \pm$ 0.02 (Fig. 5f, h). Moreover, cell viability was increased in the cotransfection group compared with the lincRNA PADNA knockdown group, the relative values are $1 \pm 0.11,0.66 \pm 0.04,0.43 \pm 0.06,0.59 \pm 0.09$ (Fig. 5g). Through rescue experiments, we proved that the lincRNA PADNA/miR-194/FBXW7 axis plays an important role in bupivacaine-induced neurotoxicity.

\section{Discussion}

The current research demonstrated that the expression of lincRNA PADNA was significantly increased in a time- and dose-dependent manner. LincRNA PADNA could prevent bupivacaine-induced neurotoxicity through the miR-194/FBXW7 axis, which may be used as a new target for inhibiting or reversing bupivacaineinduced neurotoxicity.

Anesthetic-induced neurotoxicity is one of the adverse drug reactions (ADRs) of bupivacaine that can cause permanent and irreversible neurological complications. LncRNAs have been found to play a role in anestheticinduced neurotoxicity. For example, propofol was reported to induce neuroapoptosis in the hippocampus, with differential expression of 159 lncRNAs and 100 mRNAs (fold change $\pm 2.0, P<0.05$ ) (Logan et al. 2018). In the current research, we established a bupivacaineinduced neurotoxicity model by treating DRG neurons with various concentrations of bupivacaine $(0.5,1.0,1.5$ or $2.0 \mathrm{mM}$ ) and different durations $(6 \mathrm{~h}, 12 \mathrm{~h}, 24 \mathrm{~h}$, or $48 \mathrm{~h}$ ) and we found that the expression of lincRNA PADNA was significantly increased in a time- and dosedependent manner. To further investigate the role of lincRNA PADNA, we constructed a knockdown vector to assess the function of lincRNA PADNA and found that knockdown of lincRNA PADNA significantly promoted cell death and reduced cell viability after exposure 


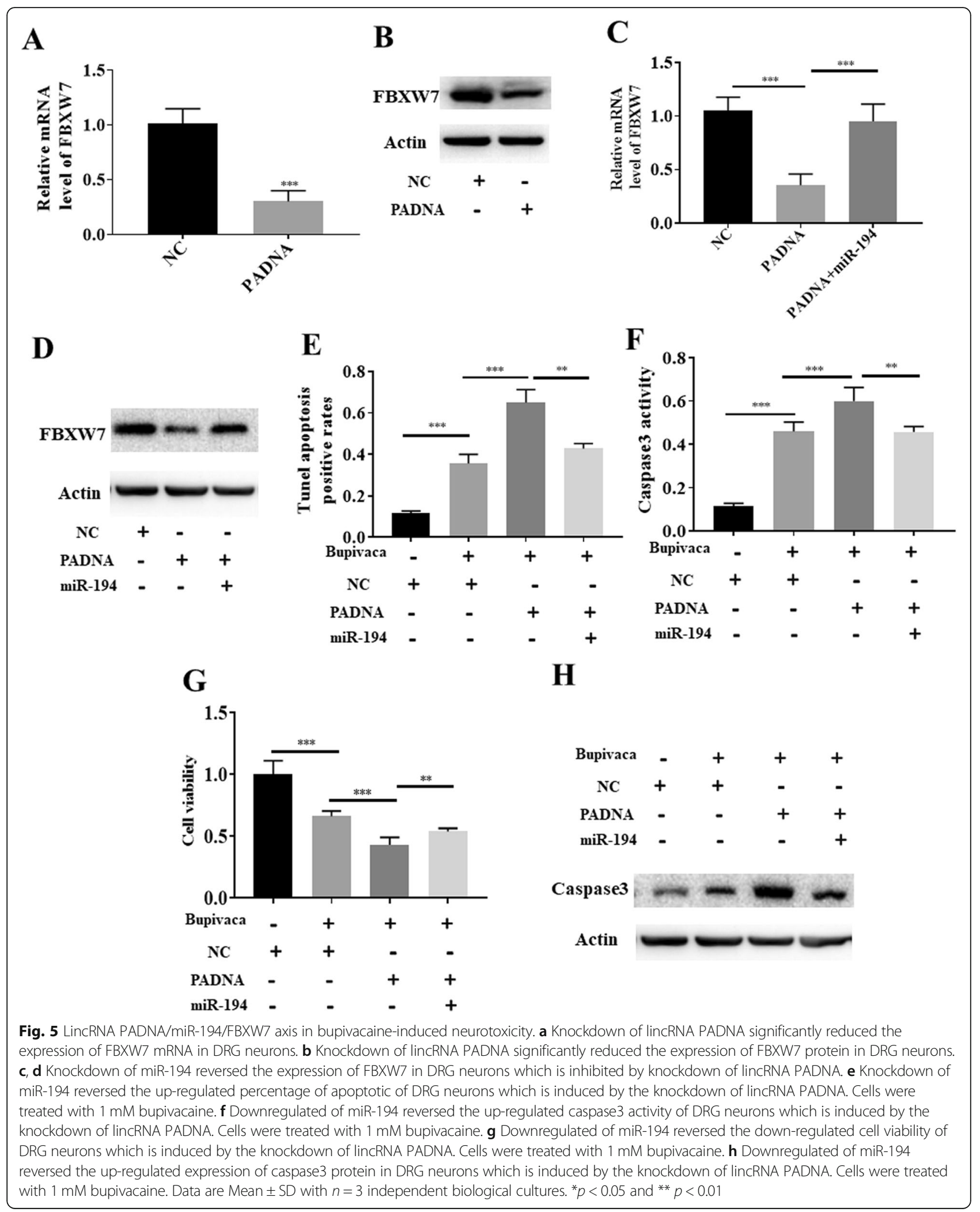


to bupivacaine. Our experiments demonstrated that lincRNA PADNA played a protective role in bupivacaineinduced neurotoxicity.

LncRNAs are extensively reported to be involved in the regulation of gene transcription (Bajolle et al. 2009), epigenetic regulation and especially posttranscriptional regulation (Ponting et al. 2009). Previous studies have reported that lncRNAs can function as competing endogenous RNAs to sponge miRNAs in various diseases (Liu et al. 2014; Luan et al. 2018). In the current research, we used bioinformatics analysis to predict the possible targets of lincRNA PADNA and found that miR-194, which has been reported to play a role in inhibiting malignant tumor progression, may be a direct target of lincRNA PADNA. Although research has indicated that miR-194 could inhibit intervertebral disc degeneration, the role of miR-194 in anesthetic-induced neurotoxicity remains unclear. To further explore the possible molecular biological function of miR-194, we first conducted a dual-luciferase reporter assay and found that the relative luciferase activity was significantly reduced in HEK cells cotransfected with the wt-lincRNA PADNA vector and miR-194 mimic compared with the mut-lincRNA PADNA group. Thus, the above results preliminarily identified miR-194 as the target of lincRNA PADNA. Next, we found that overexpression of miR194 markedly reduced the expression of lincRNA PADNA and vice versa. Moreover, we also analyzed the expression of miR-194 in bupivacaine-treated cells, and our results revealed that the expression of miR-194 was clearly reduced with increasing concentrations of bupivacaine and reached its lowest level at $2.0 \mathrm{mM}$ bupivacaine. The above results revealed that lincRNA PADNA could negatively regulate the expression of miR-194 in the setting of bupivacaine-induced neurotoxicity.

To further verify the role of the lincRNA PADNA/ miR-194/FBXW7 pathway in bupivacaine-induced neurotoxicity, we conducted a comprehensive analysis and found that the expression of FBXW7 was downregulated in the lincRNA PADNA knockdown group. Cotransfection of miR-194 and lincRNA PADNA reversed the expression of FBXW7. Rescue experiments revealed that cotransfection of miR-194 and lincRNA PADNA reduced the effect induced by PADNA.

\section{Conclusion}

Therefore, the results of comprehensive studies are in accordance with our hypothesis, and it is reasonable to believe that lincRNA PADNA could prevent bupivacaineinduced neurotoxicity by sponging miR-194 and inhibiting its function of targeting FBXW7.

\section{Abbreviations}

CNS: Central nervous system; ADRs: Adverse drug reactions; ncRNAs: Noncoding RNAs; PADNA: Protect cell death RNA; FBXW7: F-box and WD repeat domain containing 7; WT: Wide type; UTR: Untranslated region; Mut: Mutant; MTT: 3-(4,5-dimethylthiazol-2-yl)-2,5-diphenyltetrazolium bromide; TUNEL: Terminal deoxynucleotidyl transferase-mediated dUTP nick end-labeling

\section{Acknowledgements}

Not applicable.

\section{Authors' contributions}

Fan Yuning and Chen Liang performed the experiment. Wang Tenghuan analyzed the data. Nan Zhenhua analyzed the bioinformatics results. Fan Yuning wrote the manuscript. Gong Shengkai supervised the whole work. The author(s) read and approved the final manuscript.

\section{Funding}

The work is supported by the key scientific and technological projects of Henan Province (122102310223)

Availability of data and materials

The dataset used and/or analyzed during the current study are available from the corresponding author on reasonable request.

Ethics approval and consent to participate

Not applicable.

Consent for publication

All authors read and approved the final manuscript.

Competing interests

Authors declare no conflict of interest existed.

\section{Author details}

${ }^{1}$ Department of Anesthesiology, Pain and Perioperative Medicine, The First Affiliated Hospital of Zhengzhou University, Henan, China. ²Department of Anesthesiology, The Fourth Hospital of Shijiazhuang, Shijiazhuang, Hebei, China.

Received: 21 May 2020 Accepted: 3 August 2020

Published online: 13 August 2020

\section{References}

Ammar AS, Mahmoud KM. Ultrasound-guided single injection infraclavicular brachial plexus block using bupivacaine alone or combined with dexmedetomidine for pain control in upper limb surgery: a prospective randomized controlled trial. Saudi J Anaesth. 2012;6:109-14.

Bajolle F, Zaffran S, Bonnet D. Genetics and embryological mechanisms of congenital heart diseases. Arch Cardiovasc Dis. 2009;102:59-63.

Chalkiadis GA, et al. Comparison of lumbar epidural bupivacaine with fentanyl or clonidine for postoperative analgesia in children with cerebral palsy after single-event multilevel surgery. Dev Med Child Neurol. 2016;58:402-8.

Ferrillo MG. Treatment of complex regional pain syndrome with stellate ganglion local anesthetic blockade: a case report of one patient's experiences with traditional bupivacaineHCl and liposome bupivacaine. Clin Case Rep. 2016;4: $861-5$

Gu J, et al. Downregulation of IncRNA GAS5 confers tamoxifen resistance by activating miR-222 in breast cancer. Cancer Lett. 2018;434:1-10.

Guo J, et al. Antidepressant imipramine protects bupivacaine-induced neurotoxicity in dorsal root ganglion neurons through co-activation of TrkA and TrkB. J Cell Biochem. 2017;118(11):3960-7.

Helal SM, Eskandr AM, Gaballah KM, Gaarour IS. Effects of perineural administration of dexmedetomidine in combination with bupivacaine in a femoral-sciatic nerve block. Saudi J Anaesth. 2016;10:18-24.

Jiang R, Zhang C, Gu R, Wu H. MicroRNA-489-3p inhibits neurite growth by regulating PI3KNAKT pathway in spinal cord injury. Pharmazie. 2017;72:272-8.

Kurihara Y, Ikegaki J, Kato H, Chigusa S, Kaetsu H. Intrathecal segmental analgesia with a single dose of bupivacaine for cancer pain in the abdominal wall. Masui. 2008:57:182-6.

Li Y, et al. Long non-coding RNA-SNHG7 acts as a target of miR-34a to increase GALNT7 level and regulate PI3K/Akt/mTOR pathway in colorectal cancer progression. J Hematol Oncol. 2018;11:89. 
Liu M, et al. MicroRNA-29c overexpression inhibits proliferation and promotes apoptosis and differentiation in P19 embryonal carcinoma cells. Gene. 2016; 576:304-11.

Liu Y, et al. IncRNA GAS5 enhances G1 cell cycle arrest via binding to YBX1 to regulate p21 expression in stomach cancer. Sci Rep. 2015;5:10159.

Liu Z, et al. Essential role of the zinc finger transcription factor Casz1 for mammalian cardiac morphogenesis and development. J Biol Chem. 2014; 289:29801-16.

Logan S, et al. Propofol alters long non-coding RNA profiles in the neonatal mouse Hippocampus: implication of novel mechanisms in anestheticinduced developmental neurotoxicity. Cell Physiol Biochem. 2018:49:2496510

Luan W, et al. Long non-coding RNA H19 promotes glucose metabolism and cell growth in malignant melanoma via miR-106a-5p/E2F3 axis. J Cancer Res Clin Oncol. 2018:144(3):531-42.

Ponting CP, Oliver PL, Reik W. Evolution and functions of long noncoding RNAs. Cell. 2009;136:629-41.

Radwan IA, Saito S, Goto F. The neurotoxicity of local anesthetics on growing neurons: a comparative study of lidocaine, bupivacaine, mepivacaine, and ropivacaine. Anesth Analg. 2002;94:319.

Song YX, et al. Non-coding RNAs participate in the regulatory network of CLDN4 via ceRNA mediated miRNA evasion. Nat Commun. 2017:8:289.

Traore M, et al. Cauda equina syndrome and profound hearing loss after spinal anesthesia with isobaric bupivacaine. Anesth Analg. 2006;102:1863-4.

Wang JX, et al. MicroRNA-103/107 regulate programmed necrosis and myocardial ischemia/reperfusion injury through targeting FADD. Circ Res. 2015;1 17:352.

Wu X, et al. miR-194 suppresses metastasis of non-small cell lung cancer through regulating expression of BMP1 and p27(kip1). Oncogene. 2014;33:1506-14.

Xianjie W, et al. Neurotoxicity induced by bupivacaine via T-type calcium channels in SH-SY5Y cells. PLoS One. 2013;8:e62942.

Yamada A, et al. A RNA-sequencing approach for the identification of novel long non-coding RNA biomarkers in colorectal cancer. Sci Rep. 2018;8:575.

Zhang Y, Yan L, Cao Y, Kong G, Lin C. Long noncoding RNA BDNF-AS protects local anesthetic induced neurotoxicity in dorsal root ganglion neurons. Biomed Pharmacother. 2016b;80:207-12.

Zhang ZZ, et al. Long non-coding RNA LINC00628 functions as a gastric cancer suppressor via long-range modulating the expression of cell cycle related genes. Sci Rep. 2016a;6:27435.

\section{Publisher's Note}

Springer Nature remains neutral with regard to jurisdictional claims in published maps and institutional affiliations.

Ready to submit your research? Choose BMC and benefit from:

- fast, convenient online submission

- thorough peer review by experienced researchers in your field

- rapid publication on acceptance

- support for research data, including large and complex data types

- gold Open Access which fosters wider collaboration and increased citations

- maximum visibility for your research: over $100 \mathrm{M}$ website views per year

At $\mathrm{BMC}$, research is always in progress.

Learn more biomedcentral.com/submissions 